\title{
Explore Disease Mapping of Hepatitis B Using Geostatistical Analysis Techniques
}

\author{
Shaobo Zhong ${ }^{1}$, Yong Xue ${ }^{1,2, *}$, Chunxiang $\mathrm{Cao}^{1}$, Wuchun $\mathrm{Cao}^{3}$, Xiaowen $\mathrm{Li}^{1}$, \\ Jianping Guo ${ }^{1}$, and Liqun Fang ${ }^{3}$
}

\author{
${ }^{1}$ State Key Laboratory of Remote Sensing Science, Jointly Sponsored by the Institute of \\ Remote Sensing Applications of Chinese Academy of Sciences and Beijing Normal University, \\ Institute of Remote Sensing Applications, Chinese Academy of Sciences, P.O. Box 9718, Beijing \\ 100101, China \\ ${ }^{2}$ Department of Computing, London Metropolitan University, 166-220 Holloway Road, \\ London N7 8DB, UK \\ ${ }^{3}$ Institute of Microbiological Epidemiology, Academy of Military Medical Sciences, \\ Beijing, China \\ zhongshaobo163@163.com, y.xue@londonmet.ac.uk
}

\begin{abstract}
This paper presents the application of Exploratory Spatial Data Analysis (ESDA) and Kriging from GIS (ArcGIS8.3) in disease mapping through the analysis of hepatitis $\mathrm{B}$ in China. The research shows that geostatistical analysis techniques such as Kriging and ESDA have a good effect in disease mapping. Kriging methods can express properly the spatial correlation. Furthermore, unlike model-based methods, which largely depend on assumption for disease data, the Kriging method is more robust for the data. So it can be used more widely and is more operational. What's more, the Kriging method may be adapted to interpolate nonstationary spatial structure. This can expand its application more largely. At last, the Kriging method can estimate the uncertainty of prediction while many deterministic methods cannot do so. In conclusion, it is an effective operational procedure to gain a deep insight into the disease data through ESDA before mapping disease using the Kriging method.
\end{abstract}

\section{Introduction}

When we practise analysis and surveillance of diseases, the use made of maps of disease incidence are many and various. Disease maps can be used to assess the need for geographical variation in health resource allocation, or could be useful in research studies of the relation of incidence to exploratory variables (Lawson et al. 1999). Disease mapping usually chooses certain spatial interpolation method(s), and then creates a continuous surface of disease distribution according to geographically distributed sampling data of disease. There are all kinds of spatial interpolation methods, which include Inverse Distance Weighted (IDW), global polynomial, local polynomial, and Kriging etc. IDW, global polynomial and local polynomial belong to

\footnotetext{
* Corresponding author.
} 
deterministic spatial interpolation methods. This is, random effect is not taken into consideration for these methods. However, the Kriging method differs from these methods. It addresses that spatial data not only exist trend but also spatial variation. Differing from the deterministic interpolation methods, it introduces random factors and correlation factors, which are reasonable for many infectious diseases, into its activities (Carrat et al. 1992, Torok et al. 1997, Kleinschmidt et al. 2000, Croner et al. 2001). Thus, the result of disease mapping is more correct.

Exploratory Data Analysis (EDA) is an approach/philosophy for data analysis that employs a variety of techniques (mostly graphical) to insight into data itself such as uncover underlying structure, extract important variables, detect outliers and anomalies, test underlying assumptions, develop parsimonious models and determine optimal factor settings (http://www.itl.nist.gov/div898/handbook/eda/). Exploratory Spatial Data Analysis (ESDA) is EDA related to spatial data. Before Kriging the disease data, we often want to learn about the data first (e.g. whether the data exist spatial trend, which is important for selecting a proper Kriging) and we can preprocess data for meet certain needs (Johnston et al. 2001). ESDA enables us to gain a deeper understanding of disease data so that we can take correct action and make better decisions on issues relating to our data.

This paper first introduces EDA and Kriging. Then it presents an application of Kriging in disease mapping according to a case study of Hepatitis B in China. We conclude that geostatistical analysis techniques such as Kriging and ESDA have a good prospect in disease mapping. It is an effective operational procedure to gain a deep insight into the disease data through ESDA before mapping disease using Kriging methods.

\section{Materials and Methods}

\subsection{Exploratory Spatial Data Analysis Techniques}

EDA is an approach/philosophy for data analysis that employs a variety of techniques (mostly graphical) to gain deep insight into the data ( http://www.itl.nist.gov/div898/ handbook/eda/). It is different from classical analysis or Bayesian analysis in the procedure.

- For classical analysis, the sequence is:

Problem, Data, Model, Analysis, Conclusions

- For EDA, the sequence is:

Problem, Data, Analysis, Model, Conclusions

- For Bayesian, the sequence is:

Problem, Data, Model, Prior Distribution, Analysis, Conclusions.

For EDA, clearly seen from the above, the data collection is not followed by a model imposition, rather it is followed immediately by analysis with a goal of inferring what model would be appropriate. EDA tries to uncover and understand the data from the data itself. So it has unique advantages over others. 
ESDA is a special EDA, which aims at the spatial data. With ESDA, we can:

- Examining the distribution of spatial data,

- Looking for global and local outlier,

- Looking for global trend,

- Examining semivariogram/covariance and spatial structure and directional variation of the data.

In epidemiological study, all kinds of disease data hold their own characteristics (e.g. spatial structure and distribution etc.). In order to ascertain or verify these characteristics, ESDA provides us with some good approaches. Now, almost all the mainstream GIS software packages are equipped with geostatistical analysis tools. For instance, ArcGIS developed by ESRI provides Geostatistical Analyst as an extension. It includes these ESDA tools: Histogram, QQPlot, Voronoi map, Trend Analysis and Semivariogram/Covariance cloud etc (Johnston et al. 2001).

\subsection{Kriging Method}

The kriging method was put forward by a South African mining engineer D.G. Krige in 1951 and was developed by a famous French geographer G. Matheron (Matheron 1963). The method absorbs the concept of geostatistics, which addresses that any continuous properties in geo-space are quite irregular and cannot be modeled using simple smoothing mathematical function, but can be depicted properly with random surface. The property changing continuously along with the geo-space is called as "regionalized variable". It can be used in depicting continuous index variables such as air pressure, elevation and so on. Though the disease rates, unlike air pressure or elevation, holds obvious continuously distributed characteristics, health data are better examined by methods that assume that disease rates are spatially continuous (Rushton 1998). In practice, this assumption is reasonable for most widely distributed diseases.

The interpolation model of Kriging can be expressed as follows:

$$
Z(s)=m(s)+\varepsilon_{1}(s)+\varepsilon_{2}
$$

where $Z(s)$ denotes the interpolation value, which is composed of three parts: $m(s)$ reflects the spatial trend of the data, $\varepsilon_{1}(s)$ is the variation relevant to the change of spatial location $s$. And $\varepsilon_{2}$ is the residual (Gaussian noise), which has mean 0 and covariance $\sigma^{2}$ in space and has nothing to do with the change of the spatial location. $s$, which is looked on as the position expressed with $\mathrm{x}$ (longitude), $\mathrm{y}$ (latitude) ordinates, denotes the location of sampling points.

In generally, $\varepsilon_{1}(s)$ is presented with semiviaogram $\gamma(h)$ in Kriging Model. Semiviogram, only relevant to the distance $h$ of sample point pairs (this characteristic is called as stationary), reflects the effect on which the change of spatial location have and be expressed as the function of $h$. Usually, semivariogram is monofonic increasing function of $h$ and reflects the change of spatial correlation along with $h$, i.e. the closer the distance is, the stronger the spatial correlation is. Versus, the weaker the correlation is.

All kinds of Kriging models, including Simple kriging, Ordinary kriging, Universal kriging, Indicator Kriging, Probability Kriging etc., are based on the above formula (1). Each Kriging model is suited for specific situation and should be chosen seriously on purpose of best prediction. 


\subsection{Description of the Data and Processing}

China is a high prevalence area of Hepatitis B. Several nation-wide censuses of hepatitis B show the proportion of the HBsAg carriers is over $10 \%$ on average and the prevalence of $\mathrm{HBV}$ over $60 \%$. Furthermore, reported acute hepatitis cases are 2,700,000 every year according to the statistics of CDC in China. Hepatitis B has been one of the most serious problems of public health in China.

The data for this research were obtained from the statistics of Hepatitis B between $1994 \sim 1998$ when the nation-wide screening was carried out thoroughly. Thus, there is a great deal of high quality and detailed sample data. Nevertheless, west and north of China have less sample data than other places such as middle, south and east (Fig. 1) due to different administrative region size and imbalance of socioeconomic development.

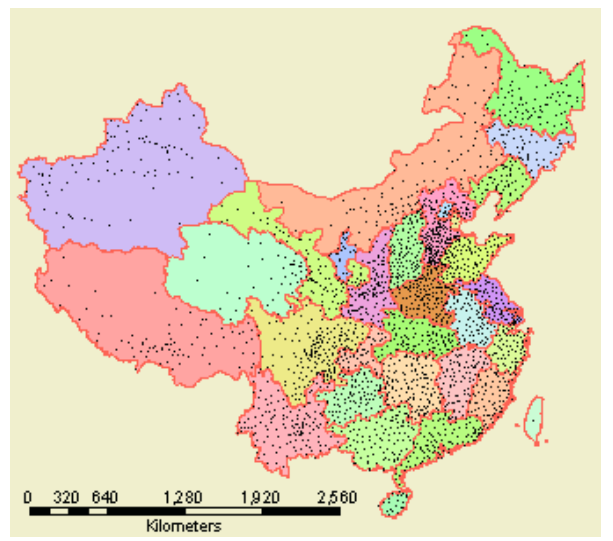

Fig. 1. The distribution of sampling points all over the nation without some islands in Southern Sea

In order to carry out the preprocessing and mapping, we have mainly collected the following data: 1) 1994-1998 county level new cases data of hepatitis B, 2) 1994-1998 county level age-grouped census and 3) 1994 screening data of hepatitis B all over the nation. We first calculate Standardized Mortality Ratio (SMR) of Hepatitis B from the above data (http://www.paho.org/English/SHA/be_v23n3- standardization.htm), then mapping SMR of Hepatitis B in China using Geostatistical techniques including ESDA, Kriging etc.

\section{Result and Discussion}

\subsection{Trend in the Data}

Trend analysis is very important for the subsequent choice of Kriging model. In ArcGIS, Trend analysis tool provides a three-dimensional perspective of the data. The locations of the sampling points are plotted on the $\mathrm{x}, \mathrm{y}$ plane. Above each sampling 
point, the value is given by the height of a stick in the $\mathrm{z}$ dimension. Especially noteworthy, the values of $\mathrm{z}$ are projected onto the $\mathrm{x}, \mathrm{z}$ plane and the $\mathrm{y}, \mathrm{z}$ plane, which makes users more easily find out the trend of the data.

Fig. 2, plotted from the disease data of 1994, shows the space distribution of the sampling points of the disease through the Trend Analysis tool. The population data are derived from the 1994 1998 censuses. The disease data were provided by Institute of Microbiological Epidemiology, Academy of Military Medical Sciences, China. From the graph, we can find out clearly that there is nonlinear trend (e.g. quadratic) from the data set. Thus, we may choose Ordinary Kriging or Universal Kriging to interpolate the data because they both can detrend the data.

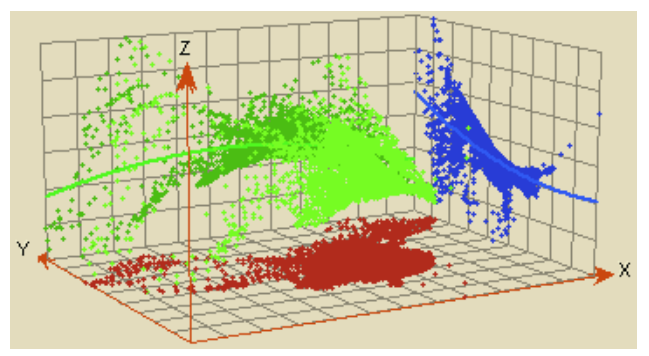

Fig. 2. Disease data of 1994 visualization in 3D space, $x, y$ represent the locations of the disease sampling points and $\mathrm{z}$ is the SMR of the disease

\subsection{Distribution of the Data Set}

Certain Kriging methods work best if the data is approximately normally distributed (a bell-shaped curve). Furthermore, Kriging also relies on the assumption of stationary. In many cases, the distribution of the original data maybe not meet the need of the Kriging model and it is necessary to transform the data (e.g. Logarithmic transformation, Box-Cox transformation and Arcsine transformation). Histogram tool of geostatistical analyst extension in ArcGIS can explore the distribution of the data and make transformation, if necessary, for the data.

Before transformation, quite obviously, the distribution has a positive skewness from the histogram of the SMR data of 1994. By performing the Box-Cox transformation and adjusting the parameter, we produce a fairly good-shaped distribution.

\subsection{Empirical Semivariogram}

Semivariogram is a key component in a Kriging model. Exploring of the semivariogram mainly is to fit its model. There are many commonly used models for semivariogram (Edward et al. 1990). Figure 3 is the empirical semivariogram of the SMR of 1994, which is plotted using ArcGIS. For a large number of data, empirical semivariogram is an effective approach to fit the semivariogram. From the graph (a) and (b), we infer that the semivariogram of the data exists obvious anisotropy. The anisotropy is presented in (a) (spherical models with different parameters) and (b) (the 
ellipse). Here, we chose the spherical model to fit the scatter points. For more information about empirical semivariogram, and fitting models, please refer to ArcGIS.

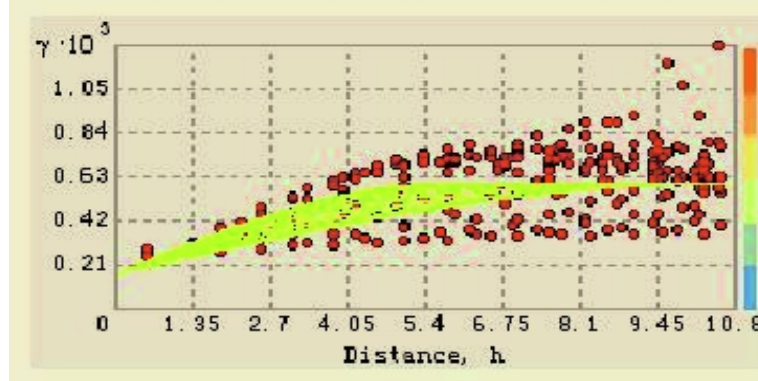

(a)

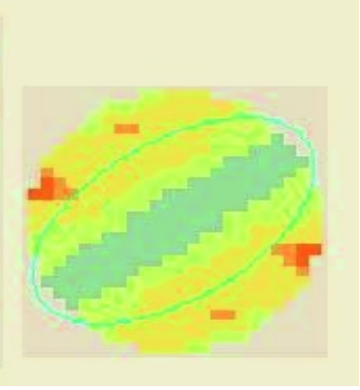

(b)

Fig. 3. Empirical semivariogram of the SMR of 1994. (a) presents different fit models changing with direction, and (b) presents the semivariogram surface

\subsection{Mapping Disease and Results Validation}

From sections 3.1, 3.2 and 3.3, finally, we can choose a proper Kriging model to interpolate the data, here Universal Kriging, semivariogram model is spherical, exists anisotropy and the data are transformed by Box-Cox with parameter 0.65 at first. With the Kriging tool from ArcGIS, we get the interpolation map. Figure 4 is produced from the data of 1994.

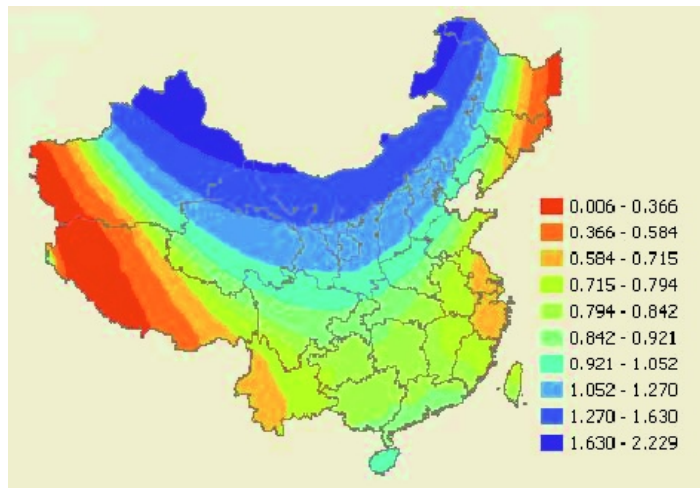

Fig. 4. SMR mapping result of hepatitis B of 1994 using Universal Kriging

When producing the result map using ArcGIS, simultaneously we get the validation result (Figure 5). The Kriging method allows us to validate the predicted values. There are two main validation methods: Cross-validation and Validation. Figure 5 is the validating result of predicted SMR of 1994 using Cross-validation. From this figure, we can see the precision of mapping is quite high. This implies that Kriging methods are suitable for the disease mapping. 


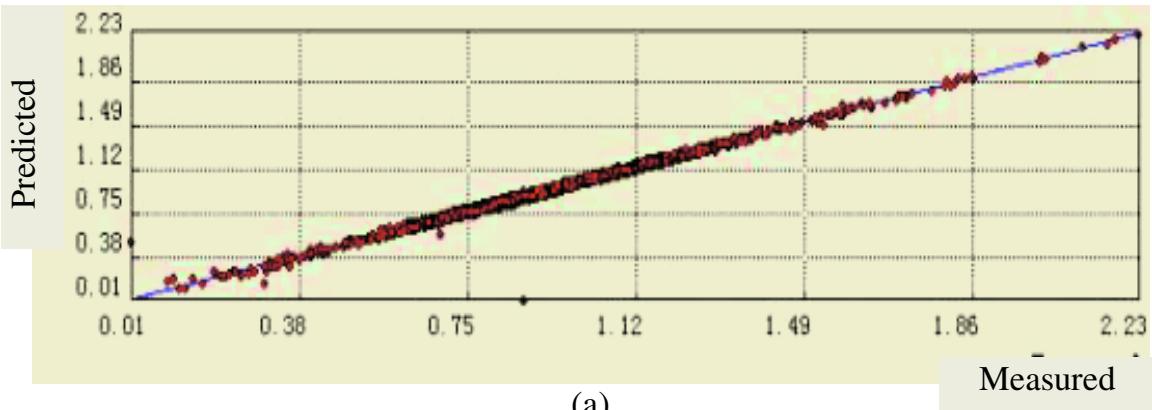

(a)

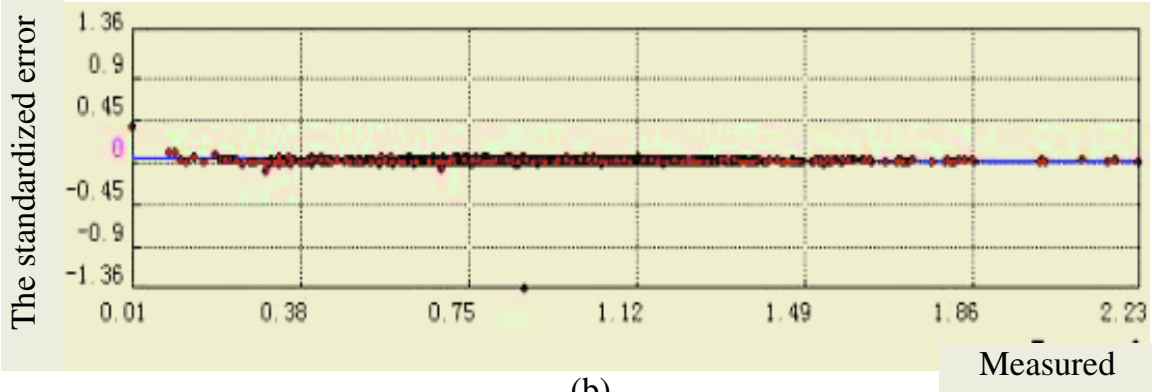

(b)

Fig. 5. Cross validation result. (a) is the comparison of predicted values and observed values and (b) is the standardized error, which is the square root of what is called Kriging variance and is a statistical measure of uncertainty for the prediction

\section{Conclusions}

Research on disease mapping covered a long history. All kinds of methods from simple ones such as point map, choropleth map and so on to complex ones like model-based methods, Kriging etc. provide analysis and surveillance of disease with strong measures. In general, complex model-based methods are more effective than the simple ones and produce more accurate results. This paper presents the application of ESDA and Kriging in disease mapping through the analysis of hepatitis B in China. The research shows that geostatistical techniques such as Kriging and ESDA have a good effect in disease mapping. Kriging methods can expresses properly the spatial correlation. Furthermore, unlike model-based methods, which largely depend on assumption for disease data (Bailey 2001), the Kriging method is more robust for the data. So it can be used more widely and is more operational. What's more, the Kriging method may be adapted to interpolate nonstationary spatial structure (Brenning et al. 2001). This can expand its application more largely. At last, the Kriging method can estimate the uncertainty of prediction while many deterministic methods cannot do so. In conclusion, it is an effective operational procedure to gain a deep insight into the disease data through ESDA before mapping disease using the Kriging method. 


\section{Acknowledgement}

This publication is an output from the research projects "CAS Hundred Talents Program" and "Innovation Project, Institute of Remote Sensing Applications" funded by Chinese Academy of Sciences. The authors wish to acknowledge related departments and individuals.

\section{References}

1. Bailey, T.C., 2001, Spatial statistical methods in health. Cad Saúde Públ, 17: 1083-1098.

2. Brenning, A., and Boogaart, K.G.v.d., 2001, Geostatistics without stationary assumptions within GIS. Proceedings of 2001 Annual Conference of the International Association for Mathematical Geology, Cancun, Mexico, September 6-12, 2001.

3. Carrat, F., and Valleron, A.J., 1992, Epidemiologic mapping using the "kriging" method: application to an influenza-like illness epidemic in France. American Journal of Epidemiology, 135(11):1293-1300.

4. Croner, C.M., and Cola, L.D., 2001, Visualization of Disease Surveillance Data with Geostatistics. Presented at UNECE(United Nations Economic Commission for Europe) work session on methodological issues involving integration of statistics and geography, Tallinn, September 2001, available at http://www.unece.org/stats/documents/2001/09/ gis/25.e.pdf.

5. Edward, H., Isaaks, R., and Mohan, S., 1990, Applied Geostatistics, Oxford University Press.

6. Johnston K., Ver Hoef, J.M., Krivoruchko, K., and Lucas N., 2001, Exploratory Spatial Data Analysis. In Use ArcGIS Geostatistical Analyst (digital book), edited by ESRI (San Diego: ESRI), pp. 81-112.

7. Kleinschmidt, I., Bagayoko, M., Clarke, G.P.Y., Craig, M., and Le Sueur, D., 2000, A spatial statistical approach to malaria mapping. International Journal of Epidemiology, 29(2):355-361.

8. Lawson, A.B., Böhning, D., Biggeri, A., Lesaffre, E., and Viel, J.F., 1999, Disease Mapping and Its Uses. In Disease Mapping and Risk Assessment for Public Health, edited by A.B. Lawson (New York: John Wiley \& Sons Ltd.), pp. 3-13

9. Matheron, G.., 1963, Principles of geostatistics. Economic Geology, 58: 1246-66.

10. NIST/SEMATECH, e-Handbook of Statistical Methods, 2004, http://www.itl.nist.gov/ div898/handbook/eda/section1/eda11.htm, accessed on November 5, 2004.

11. Rushton, G., 1998, Improving the geographic basis of health surveillance using GIS. In GIS and Health, edited by A. Gatrell and M. Loytonen(Philadelphia: Taylor and Francis, Inc.), pp. 63-80.

12. Standardization: A Classic Epidemiological Method for the Comparison of Rates, http://www.paho.org/English/SHA/be_v23n3-standardization.htm, accessed on November 5, 2004.

13. Torok, T.J., Kilgore, P.E., Clarke, M.J., Holman, R.C., Bresee, J.S., and Glass, 1997, Visualizing geographic and temporal trends in rotavirus activity in the United States, 1991 to 1996. Pediatric Infectious Disease Journal, 16(10):941-46. 